\title{
STATISTICAL PROPERTIES OF AN UNBIASED SAMPLE OF F7-K BINARIES: TOWARDS THE LONG-PERIOD SYSTEMS
}

\author{
A. Eggenberger, ${ }^{1}$ J.-L. Halbwachs,${ }^{2}$ S. Udry, ${ }^{1}$ and M. Mayor ${ }^{1}$ \\ RESUMEN
}

Hemos iniciado una investigación sobre las propiedades estadísticas de las binarias de secuencia principal, que revisa y extiende el sondeo de Duquennoy \& Mayor para estrellas de tipo solar. El analisis se divide en dos partes: las binarias espectroscópicas con períodos menores que 10 años y las binarias de largo período, incluyendo los pares visuales y los de movimiento propio común. Presentamos resultados preliminares sobre el cociente de masas intrínseco y sobre la distribución de períodos de las binarias visuales. Nuestros resultados están limitados por la estadística de números pequeños, pero combinándolos con los encontrados para las binarias espectroscópicas se obtiene los siguiente: (i) el exceso de binarias de igual masa es menos importante para los sistemas de largo período que para las binarias con $P<50$ días; (ii) la distribución de períodos es inconsistente con una distribución plana.

\section{ABSTRACT}

We have undertaken a new investigation of the statistical properties of main-sequence binaries, which is a revision and extension of the Duquennoy \& Mayor survey of solar-type stars. The analysis has been divided into two parts: the spectroscopic binaries with periods shorter than 10 years, and the long-period systems including visual binaries and common proper motion pairs. In this contribution we present preliminary results regarding the intrinsic mass ratio and period distributions of visual binaries. Our results are strongly limited by smallnumber statistics, but when combined with the ones found for the spectroscopic binaries, the following results are obtained: (i) the excess of equal-mass binaries, if still present, is less important for long-period systems than for the binaries with $P<50$ days; (ii) the period distribution is inconsistent with a flat distribution.

Key Words: BINARIES: GENERAL - BINARIES: VISUAL

\section{INTRODUCTION}

Binary stars constitute the dominant mode of star formation, but the processes underlying their formation are still not well understood. ¿From the observational point of view, some information may be gathered by studying the characteristics of binaries as a whole. For instance, the distributions of periods, eccentricities and masses (or mass ratios) may provide observational constraints for star formation models. These analyses are, however, not easy to carry out because of strong practical limitations: samples biased by selection effects, smallnumber statistics, etc.

Efforts have been made to obtain complete, volume-limited samples with known and thus corrigible selection effects that are well suited for the analysis of the intrinsic properties of binary stars. The Duquennoy \& Mayor survey of solar-type stars (Duquennoy \& Mayor 1991, hereafter DM91) used such a sample. That survey considered systems spanning a wide range of periods and some of its

\footnotetext{
${ }^{1}$ Geneva Observatory, Sauverny, Switzerland.

${ }^{2}$ Observatoire Astronomique de Strasbourg, France.
}

main results were: (1) a mass ratio distribution that is slightly increasing towards small mass ratios; (2) a log-normal period distribution with a median period of 180 years.

We have recently undertaken a revision and an extension of the DM91 survey, which makes use of the Hipparcos data and includes $\mathrm{G}$ as well as $\mathrm{K}$-dwarf stars. This study has been divided into two parts: (1) the systems with periods shorter than 10 years, which basically are spectroscopic binaries; and (2) the systems with longer periods, including visual binaries and common proper motion pairs. The analysis of the short-period systems is finished (see Halbwachs et al. 2004, and references therein) while this contribution is a presentation of preliminary results regarding the long-period part of the study. The aim of the analysis presented here is the derivation of the intrinsic mass ratio and period distributions (i.e. the distributions corrected for selection effects) of $\mathrm{G}$ and $\mathrm{K}$ main-sequence stars of the solar neighbourhood.

\section{THE HIPPARCOS UNBIASED SAMPLE}

The sample used for the analysis of the longperiod systems is larger than the one used for the 
spectroscopic binaries, since it is possible to use the completeness limit of the Hipparcos programme. Our sample is made of main-sequence Hipparcos stars with spectral types $\mathrm{F} 7-\mathrm{K}$; it is volume-limited, so only stars with a parallax $\pi>37$ mas have been retained; and only stars with a declination $\delta>-15^{\circ}$ belong to the sample. The selection of the mainsequence stars as well as the choice of 37 mas and $-15^{\circ}$ for the cutoffs are explained in Halbwachs et al. (2003). In total, 570 stars belong to our sample, which will be called the Hipparcos unbiased sample. Being volume-limited, this sample is unaffected by the bias favouring binaries with equally bright components.

\section{THE SEARCH FOR COMPANIONS}

We made an extensive search in the literature for long-period companions orbiting around any of the stars belonging to the Hipparcos unbiased sample. As we are only interested in physical systems, the following criteria have been used and considered as strong evidence that a system may be physical: (i) the system is a visual binary with a known orbit; (ii) the system is a visual pair with hint of orbital motion; or (iii) the system is a common proper motion pair. Only systems fulfilling at least one of these criteria have been kept for the analysis. These binaries have then been divided into two subsets: (1) the subset of visual binaries, defined as systems with apparent separations $0.1^{\prime \prime} \leq \rho \leq 10^{\prime \prime}$; and (2) the subset of common proper motion pairs, made of all the systems with apparent separations larger than $10^{\prime \prime}$. The preliminary results reported here concern only the subsample of visual binaries, the analysis for the common proper motion pairs still being under way.

\section{THE SELECTION EFFECTS}

In order to obtain the intrinsic distributions from the observed ones, we need to determine the selection effects affecting our sample of visual binaries. For this purpose, we need a large sample representative of our visual binaries and containing stars with the same distribution of magnitude differences as well as the same distribution of apparent separations. This sample will be called the test sample. The selection effects are determined using this test sample and will then be used for our subset of visual binaries. The test sample we are using here is made of resolved Hipparcos binaries with a spectral type earlier or equal to $\mathrm{F} 6$, or with a spectral type later than $\mathrm{F} 6$ but belonging to the main sequence.

The next step of the analysis consists in determining the detection probability for each system.
For visual binaries, this probability depends on three parameters: the apparent magnitude of the primary star $m_{1}$, the magnitude difference $\Delta m$, and the apparent separation between the two stars $\rho$. The detection probability $B\left(m_{1}, \rho, \Delta m\right)$ can therefore be written as a product of terms with all the possible degrees of crossed dependence (Halbwachs 1983):

$$
\begin{aligned}
B\left(m_{1}, \rho, \Delta m\right)= & b_{1}\left(m_{1}\right) \times b_{2}(\Delta m) \times b_{3}(\rho) \times \\
& b_{4}\left(m_{1}, \Delta m\right) \times b_{5}(\rho, \Delta m) \times \\
& b_{6}\left(m_{1}, \rho\right) \times b_{7}\left(m_{1}, \rho, \Delta m\right) .
\end{aligned}
$$

The goal is then to determine the complete detection zone, which is the region in the 3-parameter space where the detection probability $B\left(m_{1}, \rho, \Delta m\right)$ is equal to unity. Any binary will thus be considered as always detected if it belongs to the complete detection zone, and as never detected otherwise. Using our test sample, the complete detection zone for the Hipparcos resolved stars is defined by:

$$
\begin{array}{lll}
H_{\mathrm{p}} \leq 11.0, & & 0.1^{\prime \prime} \leq \rho \leq 10^{\prime \prime} \\
\Delta H_{\mathrm{p}} \leq 4.0 & \text { if } & 0.35^{\prime \prime}<\rho \leq 10^{\prime \prime} \\
\Delta H_{\mathrm{p}} \leq 10 \rho+0.5 & \text { if } & 0.1^{\prime \prime} \leq \rho \leq 0.35^{\prime \prime} \\
\Delta H_{\mathrm{p}} \leq-0.5 H_{\mathrm{p}}+8.5 & \text { if } & 9.0 \leq H_{\mathrm{p}} \leq 11.0
\end{array}
$$

Only systems fulfilling at the same time all these conditions belong to the complete detection zone. Among our 53 visual binaries with a primary star in the Hipparcos unbiased sample, 51 are within the complete detection zone and only these systems will be considered for the determination of the intrinsic distributions.

\section{THE MASS RATIO DISTRIBUTION}

The mass ratio of each of our binaries has been computed from the Hipparcos magnitudes, except for the very few systems for which a mass ratio was known from the astrometry (Martin et al. 1998; Söderhjelm 1999). This has been done by converting the Hipparcos absolute magnitudes into masses using the calibration by Harmanec (1998) and the relation by Kroupa, Tout \& Gilmore (1993). The intrinsic distribution of mass ratios has been obtained from the observed one by the method of Nested Boxes (Halbwachs et al. 1997; Halbwachs 2001). In short, this method consists of the following steps:

- For each binary, we determine its actual mass ratio, $q_{i}$, as well as $q_{\min }$, which corresponds to $\Delta H_{\mathrm{p}}^{\max }$, the maximum magnitude difference that would be allowed for this binary still to be within the complete detection zone. 


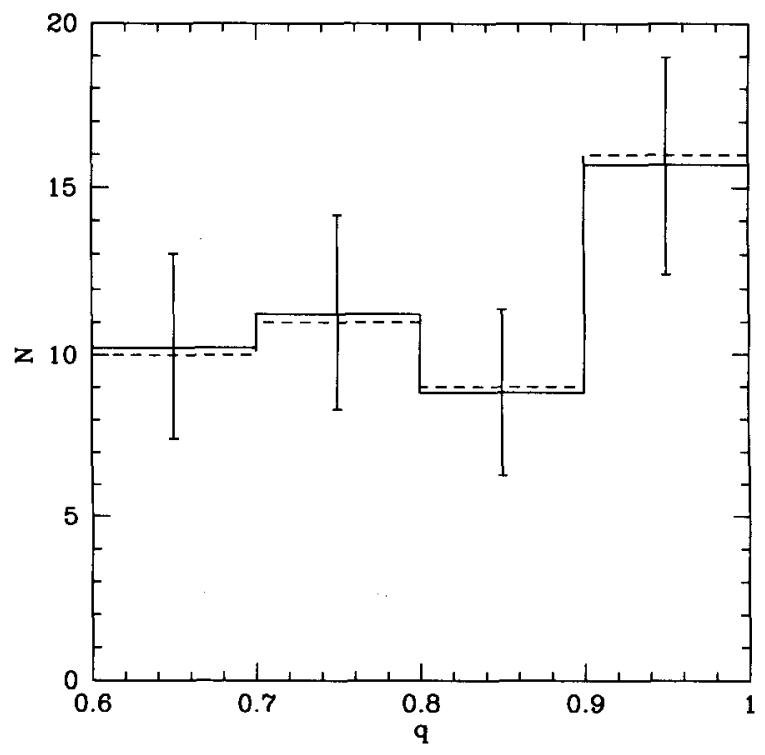

Fig. 1. Mass ratio distribution of the visual binaries with a primary belonging to the Hipparcos unbiased sample. The intrinsic distribution is represented by the solid line and the observed distribution by the dashed line.

- All the binaries with $q_{\min }<q_{i}$ can then be used to derive the partial distribution $f_{i}$ from $q=1$ to $q_{i}$.

- All the partial distributions $f_{i}$ are combined through a recurrent calculation, starting from $q=1$ and going to lower and lower mass ratios, to obtain the intrinsic distribution.

The intrinsic and observed mass ratio distributions are given in Fig. 1. The limit at $q=0.6$ comes from the condition $\Delta H_{\mathrm{p}} \leq 4.0$. The error bars have been determined by bootstrap.

We see that the intrinsic distribution of mass ratios is approximately constant, except for a small excess of systems with a mass ratio close to one, which could be due solely to statistical noise in small counts. When compared to the mass ratio distributions obtained for the spectroscopic binaries with $P<50$ days on the one hand and with $P>50$ days on the other hand (see Halbwachs et al. 2004), our mass ratio distribution is consistent with the former (agreement at the 30\% significance level), but the agreement is better with the latter distribution (98\% significance level). This result supports the observation that the excess of equal-mass binaries present for the short-period systems vanishes when systems with periods longer than 50 days are considered.

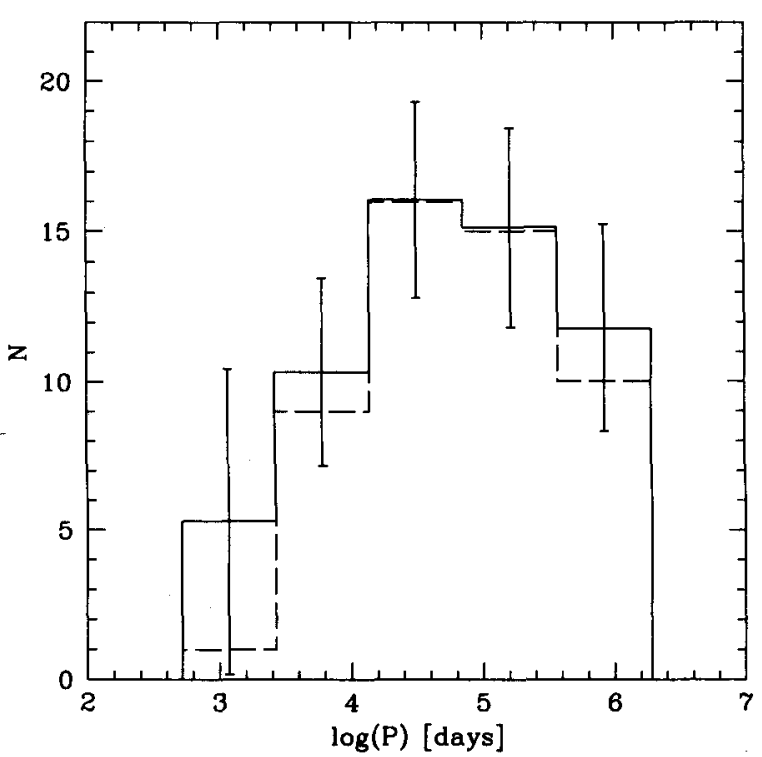

Fig. 2. Period distribution of the visual binaries with a primary in the Hipparcos unbiased sample. The intrinsic distribution is represented by the solid line and the observed distribution by the dashed line.

\section{THE PERIOD DISTRIBUTION}

For each binary, the projected separation $s$ has been computed from the apparent separation and the parallax. It has been shown (Couteau 1960) that, for visual binaries, the semimajor axis $a$ can statistically be related to the projected separation by $a=s / 0.858$. Using the mass of each component and the semimajor axis, the period has been computed by Kepler's third law.

The intrinsic distribution of periods has been obtained from the observed one by dividing the latter by the proportion of binaries detected for each value of the period. The detection probability as a function of the period can be computed as follows (Halbwachs 2001):

- For each star belonging to the Hipparcos unbiased sample, assuming it to be a binary, we let the apparent separation run in the range allowed by the boundaries of the complete detection zone. For each apparent separation, we then computed the minimum mass ratio $q_{\min }(P)$ (as a function of the orbital period when the magnitude of the primary star and the distance of the system are those of the Hipparcos star) that would be allowed for this system still to be within the complete detection zone.

- The detection probability for this hypothet- 


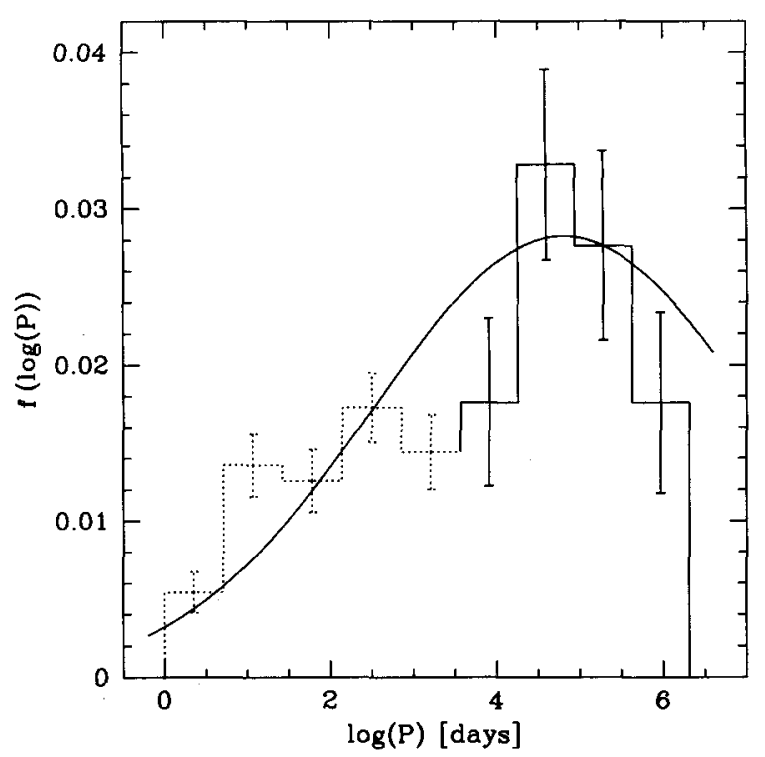

Fig. 3. Intrinsic period distribution of the spectroscopic (dotted histogram) and the visual (solid histogram) binaries with $\log (\mathrm{P}) \leq 6.31$ (results from this study and from Halbwachs et al. 2003). The curve is the log-normal law of DM91.

ical system is then given by $p_{\text {det }}^{i}(P)=$ $\int_{q_{\min }(P)}^{1} f_{\mathrm{q}}(q) \mathrm{d} q$.

- The detection probability as a function of the period is finally obtained by summing all the individual probabilities.

The observed and intrinsic period distributions are given in Fig. 2 for the visual binaries of our sample. As before, the error bars have been obtained by bootstrap. This distribution seems to exhibit a maximum consistent with the one found by DM91, but it is also compatible with a flat distribution.

Figure 3 shows the intrinsic period distribution obtained when combining our results with the ones found for the spectroscopic binaries. The log-normal law found by DM91 is also plotted on this figure. A $\chi^{2}$-test shows that the overall distribution is incompatible with a flat distribution at the $0.5 \%$ significance level, but compatible with the log-normal law. As mentioned in Halbwachs et al. (2003), the compatibility is reinforced if we consider only the spectroscopic binaries of the solar neighbourhood and discard the cluster binaries.

\section{THE BINARY FREQUENCY}

The frequency of systems with a multiplicity larger than or equal to 2 is $9.23 \%$ for the visual binaries with $0.6 \leq q \leq 1.0$ and $3.56 \leq \log (P) \leq$ 6.31. Extrapolating these statistics to smaller mass ratios using the mass ratio distribution obtained for the spectroscopic binaries with $P>50$ days, and adding the binary frequency of $13.5 \%$ found from the spectroscopic binaries with $P<10$ years, we obtain a binary frequency of $55.6 \%$ for systems with $\log (P) \leq 6.31$.

\section{CONCLUSION}

The results obtained when considering the visual binaries together with the spectroscopic binaries are:

- The intrinsic mass ratio distribution exhibits a peak of equal-mass binaries for systems with periods shorter than about 50 days. This peak gradually diminishes when longer period systems are considered and is present, but not significant, for visual binaries.

- The intrinsic period distribution is inconsistent with a flat distribution, but compatible with the log-normal law of DM91.

The results that will be obtained for the common proper motion pairs will be of great interest and will, in particular, enable us to assess the global shape of the period distribution with better accuracy.

This research has made use of the SIMBAD database and the VizieR catalogue access tool operated at CDS, France.

\section{REFERENCES}

Couteau, P. 1960, Journal des Observateurs, 43, 41

Duquennoy, A. \& Mayor, M. 1991, A\&A, 248, 485

Halbwachs, J.-L. 1983, A\&A, 128, 399

Halbwachs, J.-L. 2001, Ecole de Goutelas \#23, CNRS, 22-26 May 2000, eds. D. Egret, J.-L. Halbwachs, and J.-M. Hameury. (Societe Francaise d'Astronomie et d'Astrophysique), 35

Halbwachs, J.-L., Mayor, M., Udry, S., \& Arenou, F. 2003, A\&A, 397, 159

Halbwachs, J.-L., Mayor, M., Udry, S., \& Arenou, F. 2004, this volume

Halbwachs, J.-L., Piquard, S., Virelizier, P., Cuypers, J., Lampens, P., \& Oblak, E. 1997, ESA SP-402: Hipparcos - Venice ' 97,263

Harmanec, P. 1998, A\&A, 335, 173

Kroupa, P., Tout, C. A., \& Gilmore, G. 1993, MNRAS, 262,545

Martin, C., Mignard, F., Hartkopf, W. I., \& McAlister, H. A. 1998, A\&AS, 133, 149

Söderhjelm, S. 1999, A\&A, 341, 121 


\section{DISCUSSION}

Horch - Hipparcos has discovered many previously unknown double stars which would satisfy your parallax criterion but have little or no orbital information. If observations could be made of these stars in the coming years to determine orbital motion, would this be important for improving the results you have shown here? Eggenberger - Only a few such systems belong to our sample of 570 stars. So I do not think that the addition of these systems will change our results.

Sterzik - How many SB's are there among your sample of 53 visual binaries?

Eggenberger - There are 6 known SBs among our 53 VBs. We do not have a lot of radial velocity data for the stars belonging to the Hipparcos unbiased survey and for some stars we do not have any radial velocity measurements at all. So there may be more SBs.

Sterzik - Is your mass-ratio distribution consistent with the Duquennoy\& Mayor '91 distribution for O $<q>1$ ?

Eggenberger - We will be able to answer this question when we have the mass ratio distribution for common proper-motion pairs. Using visual binaries, we are limited to mass ratios $\geq 0.6$ and the results for common proper motion pairs will hopefully give a mass ratio distribution for mass ratios lower than this value as well. We will then compare our mass ratio distribution for VB+CPM with the one that has been obtained by DM91.

Upgren - Have you detected in your samples a minimum in the mass frequency functions for stars in general at a little less than one solar mass as noticed by Wielen and others? Have you compared the prcnerties of your stars with those of similar stars assumed to be single?

Eggenberger - We have not yet looked at that.

Anne Eggenberger, Michel Mayor and Stéphane Udry: Geneva Observatory, $51 \mathrm{Ch}$. des Maillettes, CH-1290 Sauverny, Switzerland (Anne.Eggenberger,Michel.Mayor,Stephane.Udry@obs.unige.ch).

Jean-Louis Halbwachs: Observatoire Astronomique de Strasbourg, 11 rue de l'université, F-67000 Strasbourg, France (halbwachs@astro:u-strasbg.fr). 